\title{
Improving Affective Computing Based on Semantic Analysis
}

\author{
Xue Cui ${ }^{1}$, Shi $\mathrm{Li}^{1}$ and Yiting Nan ${ }^{2}$ \\ ${ }^{1}$ College of Information and Computer Engineering, Northeast Forestry University, Harbin 150000, China \\ ${ }^{2}$ Petabase LLC, Washington, D.C. 20001, United States
}

Received 2 April 2019; Accepted 1 September 2019

\begin{abstract}
The emotional expression of normative documents, such as annual report of listed companies, is tightly bound to public interests. However, sentiment analysis in such documents is confronted with difficulties in domain adaptability, tagging, and feature extraction. In order to improve the performance of sentiment analysis for unstructured text in reports, a finegrained affective computing method based on semantic analysis was employed in this study to analyse the emotional characteristics of texts. According to the characteristics of corpus and knowledge base, the training corpus could be automatically indexed using general sentiment lexicons. The polarity of candidate emotion words in reports was distinguished via the word vector method. A sentiment lexicon oriented to the field of reports was constructed. Combined with semantic rules, a text affective computing method was proposed. The accuracy of the method was verified by experiments. Results demonstrate that the thresholds for distinguishing positive and negative reported emotional values are 0.7 and 0.5 , respectively. The expanded domain emotion words can be effectively introduced into the emotion calculation method based on semantic analysis. In contrast with the bag-of-words method used in previous studies, the proposed affective computing method based on semantic analysis can provide more accurate emotional representation of text. F value is significantly improved on the same data set. The proposed method optimizes the performance of sentiment analysis in unstructured texts.
\end{abstract}

Keywords: Text mining; Sentiment analysis; Affective computing; Annual report; Sentiment lexicon

\section{Introduction}

Sentiment analysis aims to obtain the sentiment information expressed by the publisher in the text, from which the publisher's preferences for certain things or views on social phenomena can be understood [1]. Two common methods of sentiment analysis are available [2]. The unsupervised method based on semantic analysis can calculate the emotional value of texts. Such value can reflect the intensity of emotion, and its effect depends on the choice of emotion words and the matching of semantic rules. The supervised classification method based on machine learning needs tagged corpus as the training input of the model. The most distinctive features are selected, and machine learning algorithm is used to train the corpus to obtain the classification model. The effect of these features depends on tagging and feature selection.

As a kind of official document, a report is usually a document in which a unit states its work, reflects the situation, and provides opinions or suggestions to the public or a higher organization. It has the characteristics of universality, statement, unidirectionality, and post-event. Reports usually contain factual and nonfactual information [3]. Factual information is intuitive and easy to obtain, whereas in nonfactual information, the publisher can make subjective statements according to the purpose of the report, thereby affecting the reader's judgment [4]. Unlike other sources of information, the public is more willing to believe

*E-mail address: islishi@163.com

ISSN: 1791-2377 @ 2019 School of Science, IHU. All rights reserved.

doi:10.25103/jestr.125.05 in publicly published reports. Increasing number of technical studies are keen in analyzing reports to obtain deep official information. Reports refer to long texts with limited amount, and performing tagging and feature extraction in this document is difficult, thereby restricting the development of affective computing technology.

In the existing literature, researchers have conducted studies on the sentiment analysis of reports [5-7]. However, open reports have their own characteristics of domain and tend to have limited dataset. Furthermore, the feasibility of manual tagging of the data sets is low. Despite the rapid development of sentiment analysis technology, most studies on reports are based on the statistical analysis of emotion words. However, such analysis cannot obtain sentiment information well. Therefore, accurate analysis of the sentiment of reports is an urgent problem to be solved.

This study proposes a semantic-based emotion calculation method that builds a domain sentiment lexicon based on the existing Chinese sentiment lexicon and performs semi-supervised text sentiment analysis based on semantic rules to solve the problem of domain adaptability and feature extraction.

\section{State of the art}

Sentiment analysis or opinion mining refers to the process of analyzing, processing, summarizing, and reasoning subjective texts with emotional coloring [8]. Emotional characteristics refer to the unique value information of textual data, which can express the author's opinions and attitudes through the use of words, tone, and grammar. 
Sentiment information has special values hidden in the text and may even be unknown to the author [9]. At present, researchers have performed considerable work on sentiment analysis. Xiao et al. [10] proposed a sentiment analysis strategy in Chinese microblog, which automatically expanded the domain sentiment lexicon based on the algorithm of synonym recognition and combined the degree adverb lexicon for analysis. However, the method of expanding lexicon is inefficient and exhibits poor accuracy. Jiang et al. [11] presented the semantic orientationpointwise mutual information (SO-PMI) algorithm based on Good-Turing smoothing for the problem of low coverage of emotion words in microblog in public lexicon and constructed a sentiment lexicon for microblog to determine the emotional tendency of microblog text. Given the particularity of network words, the extraction rate of candidate words in microblog is low, thereby affecting the evaluation of the experimental results. Chen [12] adopted an affective calculation method for microblog sentences that focused on the emotion words and the semantic rules of microblog texts. However, emotion words are selected through manual tagging. The personal subjectivity of artificial coders is strong that the results may be affected. Taboada et al. [13] used semantic direction calculator to determine the polarity and intensity of emotion words and to determine the emotional orientation of the text. However, the accuracy of analysis results in various fields is low. In studies on machine learning, Onan et al. [14] obtained text features using various methods and genetic algorithm to generate aggregated feature subsets. The sentiment classification result is better than that of a single feature set, but the contexts are ignored. Ghiassi et al. [15] quantified Twitter by recognizing Twitter's unique set of sentiment lexicons. Neural network and support vector machine (SVM) were used to realize the emotional classification of texts. However, the generalization ability of this experiment is weak because it is only based on Twitter. Arif et al. [16] improved the existing learning classifier system technology for short informal texts by introducing a new coding scheme to represent classifier rules to process feature vectors. The method overcomes the influence of sparse emotion words on the analysis results, but it is not suitable for long text analysis.

Financial reporting has a standardized expression but still has the characteristics of textual data. Loughran and McDonald [17] developed a financial sentiment lexicon (LM) that was suitable for English and could reduce the misclassification of sentiment analysis due to domain problems. In the studies of British annual reports, Yekini et al. [18] used the positive vocabulary frequency in LM to measure the tone of financial reports and verified the market's reaction to the enthusiasm of the annual report narrative. The technique focuses on the impact of positive vocabulary on reporting sentiment. It does not consider the negative sentiment of the report, thereby resulting in certain limitations. For American annual reports, Hjek [19] analyzed the report with positive words and negator in the report based on LM lexicon and measured emotion based on the proportion of all kinds of emotion words to the total number. After combining the emotional characteristics, the accuracy of forecasting abnormal stock return was also significantly augmented. However, considering the appearance of negative words in the report, the analysis is not comprehensive enough, and no analysis for sentiment mantic features is available. For Chinese annual reports, Xie and Lin [20] calculated the frequency of emotion words in annual reports to quantify the tone based on sentiment lexicon. The analysis of text structural features is not comprehensive enough, and the accuracy of the results based on the frequency of words is low. Song et al. [21] adopted the SVM method to predict the emotional trend of the annual report and tagged the sentences in the annual reports from different industries. The classification results showed high accuracy in positive tendency, whereas the accuracy in the negative tendency was low. The reason is that publishers are usually optimistic about the company's situation, and supervisory classification methods cannot learn the emotional characteristics of negative texts.

These studies are mainly focused on the sentiment analysis of emotional short texts, such as commentary tweets. Moreover, the performance of long texts, such as annual reports with inconspicuous emotional features, is not ideal. Most studies on the emotions of financial reports use word frequency, which does not reflect the sentiment of the report well. In addition, Chinese and English are quite different in terms of clause structure and emotional representation because of historical and cultural factors. The sentiment lexicon for the English annual report is not well adapted to Chinese. Therefore, considering the characteristics of the report texts comprehensively, this study proposes an affective computing method based on semantic analysis, which uses domain emotion words and semantic rules to quantify implicit emotions to help obtain the abstract sentiment information of the report.

The remainder of this study is organized as follows. Section 3 establishes a standard corpus for sentiment analysis and structures a domain sentiment lexicon. The emotion of text is quantified by using fine-grained affective computing method based on semantic rules. Section 4 constructs data sets through manual annotation, verifies the performance of the proposed text sentiment analysis method by comparative experiments, and discusses the application of the results. Section 5 summarizes the conclusions.

\section{Methodology}

\subsection{Construction of sentiment lexicon}

This study selected the annual report of listed companies issued by the China Securities Regulatory Commission as the research object. The public sentiment lexicon has limitations in application scope. The word vector model is constructed on the basis of Word $2 \mathrm{Vec}$, which fully considers the semantic relationship of words in context, classifies emotion words for annual reports, and expands existing sentiment lexicon.

\subsubsection{Collection and organization of data}

We get 2000 financial reports of listed companies in 2015 and 2016, which were published on Juchao Information Online, as the training corpus, and removed text-independent information, such as forms, in the financial report..

\subsubsection{Extension of domain emotion words}

The Hownet Sentiment Dictionary, National Taiwan University Sentiment Dictionary (NTUSD), and Li Jun's Chinese commendatory and derogatory Dictionary of Tsinghua University are sorted out to construct the basic vocabulary group. Considering that diverse sentiment lexicons have various construction methods, different emotional polarities of the same emotion word may be observed in several sentiment lexicons. The use of voting 
and priority rules is necessary to judge emotional polarity. When emotion words appear in three source lexicons simultaneously and if the polarity of the word in two lexicons is the same, then the polarity will be obtained. If the polarity of the words that appear in two lexicons is different, then it is set according to the authority. Compared with NTUSD and Li Jun's Chinese commendatory and derogatory Dictionary, Hownet Sentiment Dictionary is the most authoritative.

In view of the particularity of annual report texts, many proprietary words will not appear in other fields. To resolve this defect, this study proposes an automatic classification method of emotion words based on Word2 Vec and k-nearest neighbor algorithm. The annual report texts are used as corpus to train Word2 $\mathrm{Vec}$ model, and words are transformed into 100-dimensional feature vectors. The k-nearest neighbor algorithm is used to construct a binary classifier for the polarity of emotion words. To obtain as much training corpus as possible, 703 positive words and 377 negative words are extracted from the intersection of notional words and the basic sentiment lexicon of the annual report texts. The training and testing sets are divided according to the ratio of $3: 1$. When $\mathrm{K}$ is equal to 7 , the accuracy of the classification of emotion words reaches $82 \%$. Through studies on annual report texts, most of the emotion words are found to be adjectives. To increase the accuracy of the expanded emotion words, the adjectives in the annual report are selected as candidate words, and the polarity of the candidate emotion words is evaluated by the classifier to expand the basic sentiment lexicon. In addition, considering that the annual report belongs to the financial sector, the technical terms in the financial field also have emotional tendency. We expand the technical terms from the financial lexicon of Goldman Sachs and evaluated emotion polarity according to the detailed explanations. Goldman Sachs Group is an internationally renowned investment group that conducts statistical research on the financial industry. The financial lexicon of Goldman Sachs compiled and published by Goldman Sachs Group has an authoritative guiding significance. Finally, 10103 positive words and 15228 negative words are obtained. Table 1 shows the examples of new words.

Table 1. Examples of New Words

\begin{tabular}{l|l}
\hline Style & Part of the new words \\
\hline Positive words & Strong; Bullish; Bull market; Appreciation; \\
& Diversification \\
Negative words & Risk; Depreciation; Loss; Delisting; Impairment \\
\hline
\end{tabular}

Table 2. Emotional Formula Table of Semantic Rules

\begin{tabular}{l|l|l}
\hline Serial number & Combination & Formula \\
\hline 1 & Emotion word & $S=$ word $(j)$ \\
2 & Negator and emotion word & $S=(-1)^{t} \operatorname{word}(j)$ \\
3 & Degree adverb and emotion word & $S=k^{*} \operatorname{word}(j)$ \\
4 & Adverb of degree, negator and emotion word & $S=(-1)^{t} k^{*} \operatorname{word}(j)$ \\
5 & Negator, degree adverb and emotion word & $S=(-1)^{t *} k^{*} \operatorname{word}(j) * 0.5$ \\
\hline
\end{tabular}

\subsection{Text affective computing}

In addition to the investigation of emotion words, the finegrained emotional computing method based on semantic rules was also used to quantify the emotions of the text integrated with the degree adverbs and negators.

\subsubsection{Text preprocessing}

This study uses the management discussion and analysis parts of the annual report as analysis objective. The obtained annual reports are intercepted as corpus, and irrelevant information in the reports, such as tables and figures, is eliminated.

First, the corpus is segmented. Second, the names of listed companies, such as "Long Ping High-Tech" and "Lenovo Electronics," are added to the word segmentation lexicon to improve the accuracy of word segmentation. Third, stop words, which generally refer to emotionally unrelated characters, such as prepositions, pronouns, function words, and punctuation symbols, and have no actual meaning and appear frequently in the text content, are filtered. To reduce the redundancy of text data, the stop list of Harbin Institute of Technology is used.

\subsubsection{Text semantic rules}

The modification of negators can change the polarity of emotion words by analyzing the sentences in the annual report. For example, "During the reporting period, the company's overall problems are small size, weak foundation, limited ability to resist and prevent market risks, and the profitability is not strong." "Strong" is originally a commendatory term. Given the modification of the negator "not", the emotional polarity of the sentence changed from positive to negative.

A phenomenon in which more than one negator appears in a sentence in Chinese occurs. When the number of negators is odd, the sentences denote negation; when the number of negators is even, the sentences denote affirmation.

When emotion words are modified by degree adverbs, the degree of emotional inclination will change. For example, "the main business areas of company property management, parking lot operation and housing rental industry are located in Huaqiangbei, one of the most prosperous financial and commercial districts in Shenzhen City." The degree word "most" comes before "prosperity"; hence, the emotional degree of the sentence after modification is more intense.

If the emotion word is assigned to $\operatorname{word}(j)$, the weight of the negator is -1 , the number of the negator is $t$, the weight of the degree adverb is $k$, and the emotional value of the sentence is $S$, then the emotional calculation method of semantic rules is presented as follows.

\subsubsection{Affective computing based on semantic rules}

The concept of "sliding window" is introduced here. The window size is based on a clause and can be evaluated by punctuation marks in the text. When an odd number of negators appear before emotion words in the window size range, the emotional polarity of the sentence is reversed, whereas when the number of negators is even, the emotional polarity of the sentence is maintained. In the actual algorithm processing, the weight of the emotion words can be multiplied by -1 when odd negative words are used. In 
this study, 58 negators are obtained by counting the commonly used negators. For the treatment of degree words in sentences, we use Hownet's Level of Degree Words, which are divided into six levels according to different levels. Taking 0.5 as the basic weight value, the corresponding weight is given by increasing 0.5 in turn. The processing method is the same as that of the "sliding window" of negators.

The positive tendency ( $E(p o s))$ and negative tendency ( $E$ (neg) ) of the whole text can be determined by accumulating the emotional values of sentences, which can be expressed as follows:

$$
\begin{aligned}
& E(p o s)=\sum_{i \in P o s}^{n} S(i) \\
& E(n e g)=\sum_{i \in N e g}^{n} S(i)
\end{aligned}
$$

where $S(i)$ is the emotional value of a clause in text.

$E(T)$ refers to the method of measuring the intonation of text proposed by Xie and Lin [21]. The emotional value of the whole text $(E(T))$ is expressed by using the simple proportional weight summation method:

$$
E(T)=\frac{E(p o s)-E(n e g)}{E(p o s)+E(n e g)}
$$

where $-1<=E(T)<=1$; the larger the value, the greater the positive tendency of the text.

\subsection{Specific steps of affective computing}

The specific steps are as follows:

Input: annual report text corpus.

Output: emotional value of text.

Step 1) Establish a corpus of emotional analysis of standard annual reports;

Step 2) Establish a sentiment lexicon of annual reports;

Step 3) Identify sentences with emotion words in the text;

Step 4) Calculate the positive and negative emotion tendency of the text according to Table 2 and formulas (1) and (2)

Step 5) Calculate the emotion tendency of the annual report according to formula (3).

The flow chart is shown in Fig. 1.

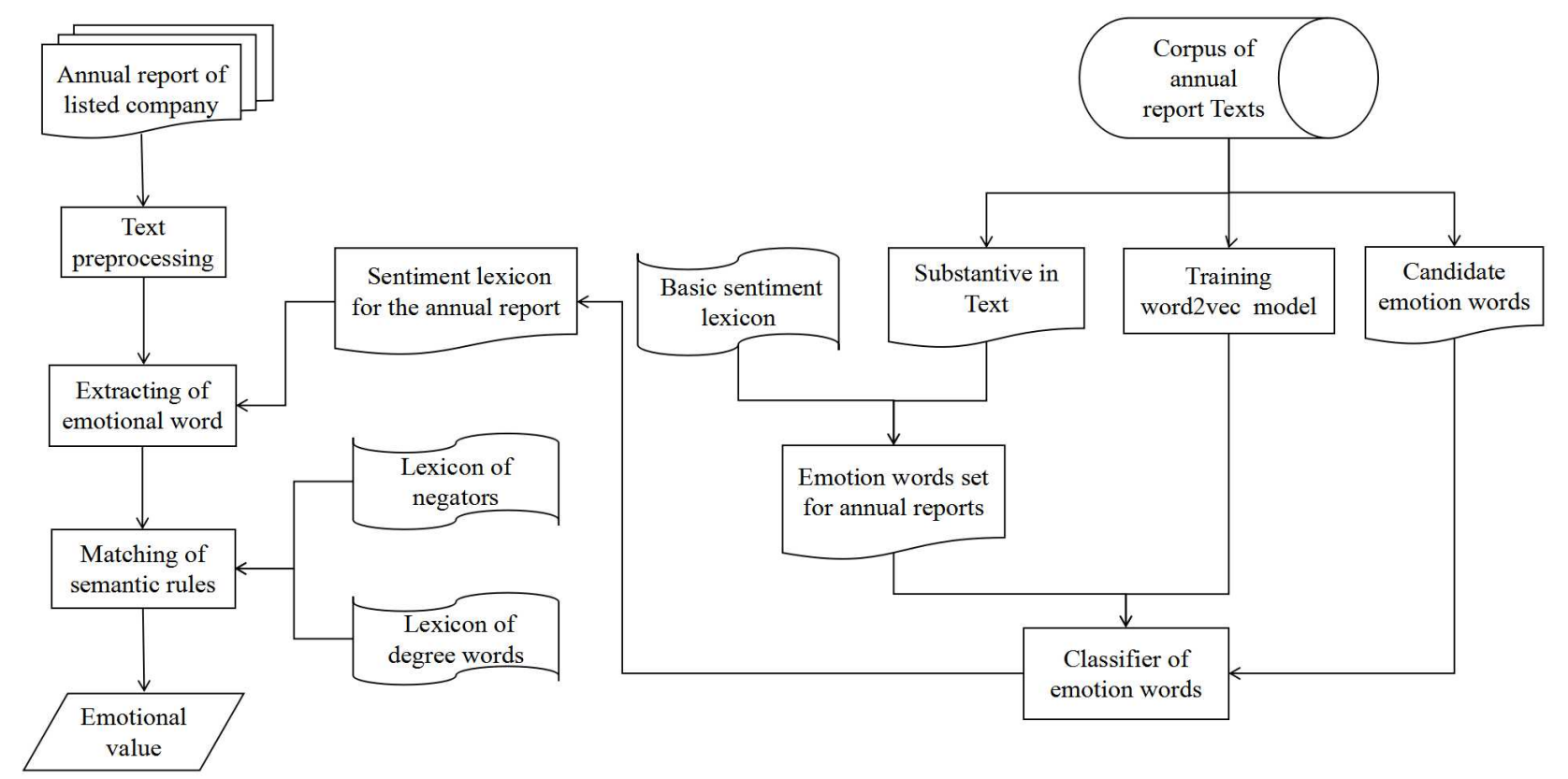

Fig. 1. Detection Steps

\section{Result analysis and discussion}

\subsection{Data Source}

The annual reports obtained by Giant Tide Information Network and 600 annual reports were randomly selected for artificial emotional tagging. Through reading the text of the annual reports, we can see that the emotions are generally positive, and only a few of the annual reports are negative. The reason is that the company's performance is poor this year and its profit is negative. Although most of the enterprises with good performance express optimistic and positive sentiment, the fact that enterprises are more optimistic about the company's development because of the decline in revenue this year due to the increase in market investment cannot be ignored. A number of enterprises only completed the annual plan, the company is in a stable state of development, and the emotional expression of the annual report is relatively stable. Therefore, considering the performance of the annual report, its sentiment is divided into three grades: positive $(\mathrm{P})$, stable $(\mathrm{S})$, and negative $(\mathrm{N})$. After the annotation of three persons familiar with field knowledge, 440 annual reports with identical annotation are selected, of which 179 are under category P, 166 are under category $\mathrm{S}$, and 95 are under category $\mathrm{N}$. The tagging results clearly show that the number of negative samples is low. This result is due to the fact that enterprises tend to convey positive signals to the outside world, which is conducive to the development of the company.

\subsection{Experimental design}

To verify the performance of the novel sentiment analysis method, a comparative experiment was conducted using the "bag-of-words" method of Xie and Lin (Method 1) and the analysis method based on general sentiment lexicon 
integrated with the semantic rules (Method 2). The method proposed in this study is referred to as Method 3. On the one hand, the design can verify the validity of the sentiment analysis method in this study when analyzing the emotions of annual reports. On the other hand, it can verify the validity of the method of expanding sentiment lexicon proposed in this study.

F-score is selected as the evaluation index of the emotion tendency. The calculation formulas are as follows:

Precision $=\frac{\text { The number of correctly classified samples of this type }}{\text { The number of marked samples of this type }}$

Recall $=\frac{\text { The number of correctly classified samples of this type }}{\text { The number of the samples of this type }}$

$F-$ score $=\frac{2 \times \text { Recall } \times \text { Precision }}{\text { Recall }+ \text { Precision }}$

\subsection{Results and analysis of experiment}

In Section 3.2, the emotional values of the annual reports are calculated. In Figure 2, the emotional value of the positive annual reports does not fluctuate considerably, and the range of 0.7 to 1 covers most positive annual reports. The range of the emotional value of stationary annual reports is from 0.5 to 0.7 , which covers the most negative annual reports. Given the differences in the expressions of various enterprises, the calculation results fluctuate greatly, and the number of annual reports that cover less than 0.5 is the largest. Therefore, the thresholds for the three types of annual reports are 0.5 and 0.7 .

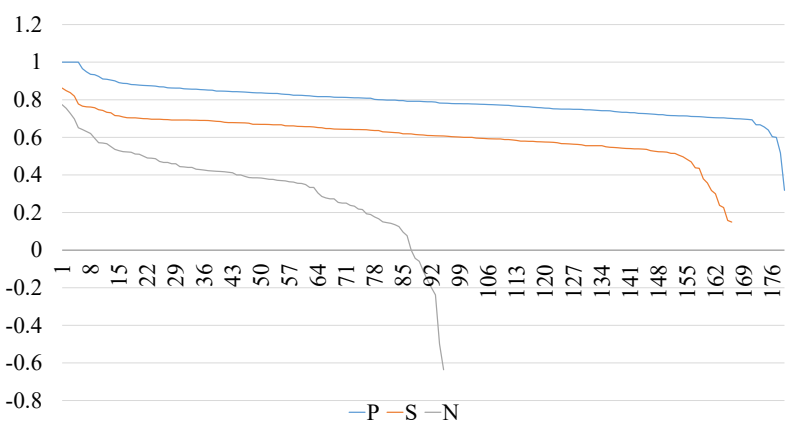

Fig. 2. Results of emotional orientation calculation.

The experimental results of the three methods are shown in Fig. 3.

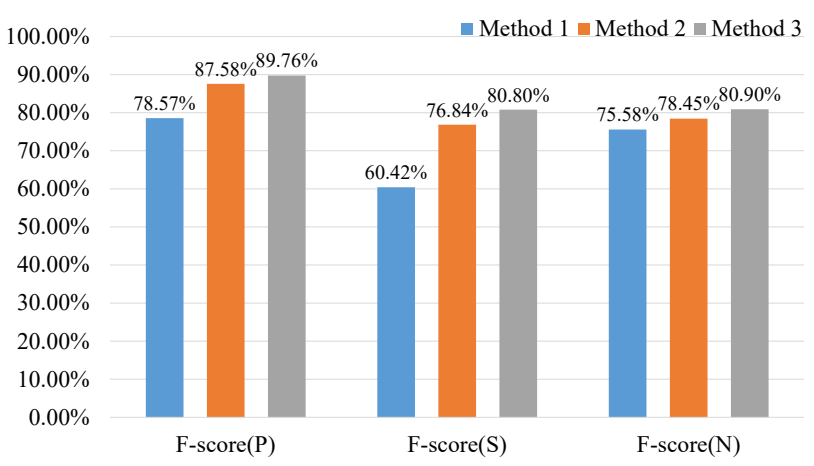

Fig. 3. Experimental results of the different methods

The performance of Method 3 was improved in all indicators, showing that the proposed method has high effectiveness and accuracy. In addition, the semantic analysis of positive annual reports is optimum. Through the analysis of the annual reports, enterprises tend to describe the positive aspects of enterprises, and fewer negative words are used to describe the difficulties faced by enterprises. These findings are consistent with the results in Song et al. [21].

\subsection{Discussion on the application of results}

As a fact-based open text, the report has the characteristics of post-event. To investigate whether publishers intentionally manipulate reports and convey false sentiment information to the public, this study conducted a significance test on the sentiment information of fraudulent and qualified listed companies.

\subsubsection{Data collection and preparation}

The list of companies published by CSMAR, Shenzhen Stock Exchange, and Shanghai Stock Exchange in 2016 and 2017 was collected to discover information about fraudulent enterprises. Considering that most of the frauds are identified by the artificial modification of key indicators (structured data), the application of unstructured data in fraudulent judgment must rely largely on the subjective opinions or empirical basis of the judges. To increase the accuracy of the empirical results, we excluded the fraudulent enterprises caused by nonverbal emotions. The criterion for screening fraudulent enterprises is that the types of violations are false records and misleading statements. A total of 85 fraudulent annual reports of 63 enterprises are used as data sets of fraudulent texts. These financial reports were obtained from the official website of the listed companies. The present study adopts the method of matching samples and selects the same number of annual reports of qualified companies to match the size, industry, and time period of the company. Finally, 85 and 83 annual reports of fraudulent and non-fraudulent enterprises were obtained, respectively. All selected annual reports are original and are not from repetitive or revised annual reports. The matching criteria are presented as follows:

(1) Firm size matching is measured by total assets. If the total assets are within the scope of $30 \%$ of the fraudulent companies in the first fraudulent year, then non-fraudulent companies are considered similar [22].

(2) The industry codes of the listed companies, which stipulates the classification and code of the whole social and economic activities and divides the national economic industries into four levels: category, large category, medium category, and small category, are used to match industries according to the National Economic Industry Classification standard issued by the state. The code consists of a Latin alphabet and four Arabic numerals, which are matched according to industry similarity. 
(3) The annual reports in the same year are selected as non-fraudulent enterprise.

\subsubsection{Significance test}

Significance test can reflect the overall characteristics according to the sample data to judge whether the difference is caused by accidental fluctuations or real differences. Before conducting the significance test, normal distribution test should be carried out on the data. The results are shown in Table 3.

Table 3. Results of Normality Test

\begin{tabular}{l|l|l|l|l|l|l}
\hline & \multicolumn{3}{l|}{ Kolmogorov-Sminov ${ }^{\text {a }}$} & \multicolumn{2}{l}{ Shapiro-Wilke } \\
\hline & Statistics & Freedom & Significance & Statistics & Freedom & Significance \\
\hline Fraud & 0.182 & 85 & 0.000 & 0.864 & 85 \\
Non-fraud & 0.130 & 83 & 0.001 & 0.853 & 0.000 \\
\hline Note: a. Riley' s significance correction \\
\hline
\end{tabular}

The results show that the significance is less than 0.05 . Thus, this value does not conform to the standard normal distribution. Nonparametric test (rank sum test) is performed on variables that do not conform to normal distribution, and the confidence level is $95 \%$. The results are shown in Table 4.

Table. 4. Results of Rank Sum Test ${ }^{\mathrm{a}}$

\begin{tabular}{l|l}
\hline & $E(T)$ \\
\hline Mann-Whitney U & 2840.500 \\
Wilcoxon W & 6495.500 \\
$Z$ & -2.180 \\
Progressive significance (two-tailed) & 0.029 \\
\hline \multicolumn{2}{l}{ Note: a. Grouping variables: whether fraudulent or not. }
\end{tabular}

Table 4 shows that the significance of rank sum test is 0.029 less than 0.05 , indicating that a significant difference exists in the emotions exhibited by fraudulent and qualified annual reports. As an afterthought document, report manipulation is usually a purposeful strategic deception that is confirmed by the interpretability of emotional changes. Therefore, the analysis of the emotional expression of such texts is of practical significance.

\section{Conclusions}

A fine-grained affective computing method based on expanded domain emotion words and text semantic rules was developed to analyze and improve the extraction of sentiment information hidden in the report. The following conclusions could be drawn:
(1) Quantified text emotional value can reflect the emotional tendency of the report, in which values higher than 0.7 , less than 0.5 , and between 0.7 and 0.5 are generally regarded as positive, negative, and emotionally stable reports, respectively.

(2) The addition of domain emotion words can optimize the performance of affective computing based on the semantic analysis of annual reports.

(3) Semantic rule matching can improve the accuracy of calculating emotions in annual reports.

This study proposes an affective computing method based on semantic analysis considering the domain and linguistic characteristics of report texts. The post-event characteristics of reports determine whether the publisher manipulated the qualitative information disclosure report. Valuable information is provided for the public by analyzing the sentiment information transmitted in the report. However, the selection of emotion words, which is affected by parts of speech, is not comprehensive enough when expanded. Therefore, a more comprehensive and accurate method of expanding emotion words in future studies should be considered.

\section{Acknowledgement}

This work was supported by the Fundamental Research Funds for the Central Universities (Grant No. 2572019BH03).

This is an Open Access article distributed under the terms of the Creative Commons Attribution License

\section{References}

1. Cambria, E., "Affective computing and sentiment analysis". IEEE Intelligent Systems, 31(2), 2016,pp.102-107.

2. Zhang, S., Wei, Z., Wang, Y., Liao, T., "Sentiment analysis of Chinese micro-blog text based on extended sentiment lexicon". Future Generation Computer Systems, 81(1),2018, pp.395-403.

3. Goel, S., Uzuner, O., "Do sentiments matter in fraud detection? Estimating semantic orientation of annual reports". Intelligent Systems in Accounting Finance \& Management, 23(3),2016, pp.215-239.

4. Ekman, P., Friesen, W.V., "Felt, false, and miserable smiles". Journal of Nonverbal Behavior, 6(4), 1982, pp.238-252.

5. Peng, M., Wang, Q., Huamg, J., Zhou, L., Hu, X., "Stock research reports classification based on sentiment analysis". Journal of Wuhan University(Natural Science Edition), 61(2), 2015, pp.124130 .
6. Hájek, P., Olej, V., Renata, M., "Forecasting stock prices using sentiment information in annual reports-a neural network and support vector regression approach". WSEAS Transactions on Business and Economics, 4(10), 2013, pp.293-305.

7. Pagliarussi, M. S., Aguiar, M. O., Galdi, F. C., "Sentiment analysis in annual reports from Brazilian companies listed at the BM\&FBovespa". Base-Revista de Administração e Contabilidade da Unisinos, 13(1), 2016, pp.53-64.

8. Zhao, Y. Y., Qin, B., Liu, T., "Sentiment analysis". Journal of Software, 21(8), 2010, pp.1834-1848.

9. Lan, Q. J., Ma, C. Q., "Text perception-new development in financial research". Journal of Changsha University of Science and Technology (Natural Science), 26(3), 2011, pp.37-40.

10. Xiao, J., Ding, X., He, R. J., "Analysis of Chinese micro-blog emotion which based on field of emotional dictionary". Electronic Design Engineering, (12), 2015, pp.18-21. 
11. Jing, L. L., He, Z. H., Zhang, H., "Research on the construction of Micro-blog sentiment lexicon based on good-turing smoothing SOPMI algorithm". Modern Computer, 610(10), 2018, pp.17-22.

12. Chen, G. L., "Microblog sentiment analysis basing on emotion dictionary and semantic rule". Information Research, (2), 2016, pp.1-6.

13. Taboada, M., Brooke, J., Tofiloski, M., Voll, K. D., Stede, M., "Lexicon-based methods for sentiment analysis". Computational Linguistics, 37(2), 2011, pp.267-307.

14. Onan, A., Korukoglu S. "A feature selection model based on genetic rank aggregation for text sentiment classification". Journal of Information Science, 43(1), 2017, pp.25-38.

15. Ghiassi, M., Lee, S., "A domain transferable lexicon set for twitter sentiment analysis using a supervised machine learning approach". Expert Systems with Applications, 106(1), 2018, pp. 197-216.

16. Arif, M. H., Li, J., Iqbal, M., Liu, K., "Sentiment analysis and spam detection in short informal text using learning classifier systems". Soft Computing, 22(21), 2018, pp.7281-7291.
17. Loughran, T., Mcdonald, B., "When is a liability not a liability? Textual analysis, dictionaries, and 10-ks". Journal of Finance, 66(1), 2011, pp.35-65.

18. Yekini, L. S., Wisniewski, T. P., Millo, Y., "Market reaction to the positiveness of annual report narratives". The British Accounting Review, 48(4), 2015, pp.415-430.

19. Petr Hájek., "Combining bag-of-words and sentiment features of annual reports to predict abnormal stock returns". Neural Computing \& Applications, 29(7), 2017, pp.1-16.

20. Xie, D. R., Lin, L., "Do management tones help to forecast firms' future performance: A textual analysis based on annual earnings communication conferences of listed companies in China". Accounting Research, 2(1), 2015, pp.20-27.

21. Song, Y., Wang, H., Zhu, M., "Sustainable strategy for corporate governance based on the sentiment analysis of financial reports with CSR". Financial Innovation, 4(1), 2018, pp. 2.

22. Larcker, D. F., "Detecting deceptive discussions in conference calls". Social Science Electronic Publishing, 50(2), 2010, pp.495540 . 